\title{
Inclusion/Exclusion Criteria Not Met Supplemental Qualifiers Dataset
}

National Cancer Institute

\section{Source}

National Cancer Institute. Inclusion/Exclusion Criteria Not Met Supplemental Qualifiers

Dataset. NCI Thesaurus. Code C147218.

A dataset containing supplemental information, specifically non-standard variables, to parent records in the inclusion/exclusion criteria not met domain. 